\title{
Advantages and disadvantages of phonological similarity in serial recall and serial recognition of nonwords
}

\author{
ARILD LIAN and PAUL JOHAN KARLSEN \\ University of Oslo, Oslo, Norway
}

\begin{abstract}
The phonological similarity effect (PSE) was studied with lists of nonwords in one task of serial recall and one task of serial recognition. PSE was critically affected by the scoring procedure and the type of phonological similarity involved, and the effect diverged in several ways from the findings of previous studies on words. PSE was absent in serial recall, regardless of scoring procedure, when phonologically similar items that shared the midvowel were compared with phonologically distinct items. PSE was reversed when serial recall and item recall scores of rhyme items and consonant frame items were compared with distinct items, but it was present in the position accuracy score of rhyme lists. In serial recognition, PSE was absent when rhyme lists were compared with distinct lists. Recognition was better for consonant frame lists than for rhyme lists, and there was a marginally significant reversal of PSE when consonant frame lists were compared with distinct lists. In the view of Fallon, Groves, and Tehan's (1999) study and the present study, rhyming improves item recall and serial recall but diminishes position accuracy, regardless of lexicality. But consonant frame lists with differing midvowels have higher item recall, serial recall, and position accuracy scores than do rhyme lists.
\end{abstract}

The phonological similarity effect (PSE) in immediate serial recall (ISR) refers to the finding that phonologically similar items, such as mad cap cat, generally are more difficult to recall in the correct order than are phonologically distinct items, such as cow bar pen. This effect was first reported by Conrad and Hull (1964) and has since been widely acknowledged in the experimental literature (Baddeley, 1986; Brown \& Hulme, 1995; Burgess \& Hitch, 1992; Longoni, Richardson, \& Aiello, 1993; Nairne, 1990; Schweickert, 1993; Schweickert, Guentert, \& Hersberger, 1990). Nairne and Kelley (1999) considered PSE to be a benchmark fact of ISR, Gathercole (1997) referred to PSE as one of the key empirical findings that affords a challenge to any model of short-term memory, and Page and Norris (1998) argued that an explanation of PSE is an essential test of such models.

PSE has been documented with a wide range of materials and procedures that are likely to activate semantic and lexical long-term memory representations. These include the use of letters (Conrad \& Hull, 1964), familiar words (Baddeley, 1966), pictures (Hitch \& Halliday, 1983), auditory presentation (Crowder, 1979; Watkins, Watkins, \& Crowder, 1974), visual presentation (Besner \& Davelaar, 1982), and paired-associate learning (Papagno \& Vallar,

This work was supported by a grant from the Norwegian Research Council to the first author. Correspondence concerning this article should be addressed to A. Lian, Institute of Psychology, Box 1094, University of Oslo, N-0317 Oslo, Norway (e-mail: arild.lian@ psykologi. uio.no).
1992). Verbal stimuli that are likely to be perceived as less meaningful or less familiar and that could be interpreted as being less well connected to semantic and lexical longterm memory have not been explored as systematically as other materials have been in the ISR task. Even the most meaningless verbal utterances are likely to activate a variety of long-term memory representations that guide perception and behavior, but the associative value of nonword utterances will generally be lower than that of regular words. That is, the participant will generally need more time to come up with an association to a nonword than to a word (Lian, Karlsen, \& Winsvold, 2001).

A consonant-vowel-consonant (CVC) combination constitutes a nonword when it does not currently form a whole word in the natural vocabulary of the participant. Nonwords differ with respect to wordlikeness in the sense that they may sound more or less like familiar words and may contain more or less the same phoneme segments as familiar words do. Phonemic segments, lexemes, meanings, and associations are long-term memory representations that people may rely on in order to retain all types of verbal material in primary memory tasks. The functional memory code may differ from case to case, depending on the familiarity and meaningfulness of the material to be remembered, the time available for processing, and other factors (Bartlett, 1932; Neisser, 1982).

Lian et al. (2001) used a reaction time measure to distinguish between nonwords of high and low associative value. All nonwords fulfilled the phonotactical rules of the first language of the participants. Yet a classical PSE was found only for nonwords with high associative value. For 
nonwords of low associative value, there was a reversal of PSE. The reversal of PSE for nonwords was recently replicated with new material and a rhyming component not present in the initial study (Lian, Karlsen, \& Eriksen, 2004). The way in which PSE interacts with lexicality and associative value implies that PSE depends on the activation of familiar lexical representations, but the reversal seems to contradict two other reports in the literature. Besner and Davelaar (1982) found that PSE occurs for nonword syllables independent of the wordlikeness of the items, and Gathercole, Pickering, Hall, and Peaker (2001) demonstrated PSE for words as well as nonwords. Yet Gathercole et al. also found an interaction between lexicality (words vs. nonwords) and phonological similarity in the form of a diminution of PSE for nonwords relative to words. Lian et al. (2001) suggested that the definition and selection of nonwords influenced those results, arguing that Besner and Davelaar's nonwords may have been of high associative value and that Gathercole et al.'s nonwords may have been a mixture of high and low associative values, with a majority being items of high associative value.

The interaction between lexicality and phonological similarity and between associative value and phonological similarity challenges all current models of phonological working memory. Note that this interaction is demonstrated by way of the traditional serial recall score- that is, the number of items recalled in correct position in the ISR task. Current models, however, account for PSE in terms of probabilities of transposition errors. The primacy model of Page and Norris (1998), which assumes a twostage choice process in the ISR task, locates the disruptive effects of phonological similarity at the output stage. A phonologically nonconfusableitem that is forwarded from the first stage will activate no items other than itself, whereas a confusable item will activate a number of other items at the output stage and thereby increase the likelihood of transposition errors. In the Burgess and Hitch (1999) model, PSE is similarly accounted for in terms of a second-stage competition among confusable items that increases the probability of transposition errors. However, the disadvantage of phonological similarity, as demonstrated by the error analysis of the recall protocol, is not necessarily associated with a similar disadvantage in relation to the serial recall score. Serial recall of phonologically similar lists may be disadvantaged in terms of transposition errors and yet be excellent in terms of the serial recall score when compared with the recall of phonologically distinct lists. Lian et al. (2004) demonstrated that transposition errors were more frequent in the recall of phonologicallysimilar CVC trigrams than in lists of phonologically distinct items. Even so, significantly more items in the former lists, as compared with the distinct lists, were recalled in the correct position. The dominance of transposition errors with the phonologically similar items did not prevent a reversal of PSE with nonword trigrams.

Fallon, Groves, and Tehan (1999) argued that the serial recall score is affected both by position accuracy and item memory, and therefore, they suggested three alternative scoring procedures for the ISR task. In addition to the commonly used (1) serial recall score (i.e., the number of items recalled in correct serial position), they introduced the (2) item score, the number of items recalled from the list regardless of the order in which they were recalled. Finally, they suggested a (3) score of position accuracy, which is the number of items recalled in correct serial position as a proportion of the number of items recalled regardless of position. Although these scoring procedures do not permit a complete disentanglement of item and order memory in the ISR task (see Neath, 1999), they do permit a structurally more complex description of the serial recall performance. First of all, it is now possible to demonstrate that phonological similarity may have different effects on serial recall performance as assessed by the three scoring criteria. The traditional serial recall score is confounded by the item recall score; the position accuracy score less so. In other words, PSE may have different effects on serial recall score and position accuracy (see Karlsen \& Lian, 2004). Therefore, we do not advise an assessment of PSE on the basis of serial recall score and transposition errors alone. Transposition errors are commonly defined as correctly recalled items in incorrect positions, and it follows that item recall is the sum of the serial recall score and the proportion of transposition errors. It also follows that the proportion of transposition errors will be inversely related to position accuracy. But as long as the number of intrusions and omissions are unknown, transpositions predict neither the serial recall nor the item scores in the ISR task.

The interaction between phonological similarity and lexicality is surprising in light of many current models of short-term memory, and it points to a distinction between two types of phonological representations. Such a distinction contrasts with the idea of a single phonological store in the working memory model (Baddeley, 2001) and with the idea of one level of acoustic features in the feature model (Nairne, 1990). Currently, it is only the interactive model by Gathercole and Martin (1996) that explicitly distinguishes between familiar and unfamiliar phonological utterances in the serial recall task. Familiar sounds may activate lexical-phonological representations in a higher level phonological space that is associated with the classical PSE, whereas unfamiliar sounds may rely more on unstable activations in the lower level phonological network that is associated with the reversal of PSE.

Other models of short-term memory may be able to account for the interaction between lexicality and phonological similarity by introducing a parameter that accounts for the lexical status of items to be remembered. Each presented word should activate the corresponding long-term memory word representation, whereas an unfamiliar trigram should activate long-term memory representations of its phonological segments. Such a parameter would account for the typical main effects of lexicality: word frequency and wordlikeness in the serial recall task (Gathercole et al., 2001; Hulme, Roodenrys, Brown, \& Mercer, 
1995). Whereas a reduced serial recall score is associated with an increase in transposition errors in the case of phonologically similar words, it is also the case that the shared phoneme segment may work as a phonological category cue that increases the item recall score of similar words. Indeed, in Lian et al. (2004) this category cuing effect is particularly strong in the case of nonwords. A difference in the item recall of distinct and similar lists, then, would account for the difference in the serial recall scores (see Karlsen \& Lian, 2004). Thus a reversal of PSE may be associated with an advantage of item recall from the phonologically similar lists, whereas the normal PSE occurs when items in the phonologically similar and distinct lists have an equal probability of being recalled.

Phonological similarity, having different effects on words and nonwords, may also affect the three types of scores in the ISR task differently. Fallon et al. (1999) argued that in some experimental conditions, phonological similarity has opposite effects on item and position accuracy scoring. In their study, item scores for three phonological categories differed significantly from each other in the following order: rhyme $>$ distinct $>$ similar nonrhyme words. There was no difference between the correctin-position scores of distinct and rhyming words, but position accuracy for distinct words was significantly higher than position accuracy for both rhyming and similar nonrhyming words. Thus, detrimental effects of phonological similarity are more evident in terms of position accuracy than in correct-in-position scores in serial recall tasks. Fallon et al. argued that the lack of a difference between the correct-in-position scores of rhyme and distinct lists is caused by phonological similarity's affecting the rhyme lists in two opposing ways: The positive effect of category cuing in item recall masks the detrimental effect of phonological similarity in recall accuracy.

Experiment 1 was designed to replicate Fallon et al.'s (1999) work using meaningless CVC trigrams instead of words. If words and nonwords are represented in different phonological layers (Gathercole \& Martin, 1996), differing with respect to the stability of representations, it may be questioned whether Fallon et al.'s finding extends to nonwords. To test this possibility, we created lists of nonwords corresponding to Fallon et al.'s three phonological categories: distinct, rhyme, and similar nonrhyme. Fallon et al.'s rhyme lists consisted of consonant-contrasting words such as cat and rat; in the present work they were consonant-contrasting nonwords such as tiv and div. In addition, we introduced a fourth category of trigrams that shared the initial and final consonants (e.g., keb and $k a b$ ), and this category of vowel-contrasting items shall be referred to as consonant frame items.

In Fallon et al.'s (1999) Experiment 1, which contained no item repetitions across conditions, position accuracy appeared to be inversely related to the number of phonemes shared by all items in the presented list. This finding can be accounted for by the primacy model of Page and Norris (1998), the Burgess and Hitch model (1999), and Nairne's (1990; Neath, 1999) feature model. However, the relationship between the number of shared phonemes and position accuracy was not demonstrated in their Experiment 2 , in which a closed set of study items was used. Therefore, the observed association between the two variables seems to be grounded on the condition that all lists include only new items. In the present Experiment 1, like in Fallon et al.'s Experiment 1, we made use of an open set of trigrams - that is, new items were presented in every trial. Moreover, the rhyme and consonant frame lists consisted of items that shared two phonemes. Hence, position accuracy for items in these categories was expected to be inferior both to position accuracy for items in the similar nonrhyme category, which share one phoneme, and to position accuracy for items in the phonologically distinct category, which share no phonemes. If there were an inverse relationship between position accuracy and the number of shared phonemes, one would expect no difference between the position accuracies of rhyme and consonant frame lists.

Surprenant and Neath (1996) found that serial recall performance for vowel-contrasting lists (consonant frame trigrams), as measured by the correct-in-position score, was better than recall performance for consonant-contrasting lists (rhyme trigrams). However, a strict examination of their procedures shows that they did not run a serial recall experiment; rather, they inferred recall on the basis of data from a serial reconstruction task, and they did not compute a position accuracy score for these lists. Thus, it remains to be seen whether and in what respect ISR performance differs for consonant frame and rhyme nonwords. Lian et al. (2004) demonstrated that recall of consonant frame nonwords is superior to recall of rhyme nonwords, in terms of both item and position accuracy, when a closed set of study items was used. This finding does not support the hypothesis that position accuracy is inversely related to the number of shared phonemes in the presented items. It also challenges most models of short-term phonological memory. However, the observed recall difference between consonant frame and rhyme nonwords may be interpreted as a processing difference between vowel- and consonantcontrasting sounds. The present study will show whether the recall superiority of consonant frame nonwords can be replicated in comparison with the three other phonological categories of rhyme, similar nonrhyme, and distinct nonwords. Also, we will test Fallon et al.'s (1999) category cuing hypothesis by examining whether the superior item recall of rhyme words in their study extends to rhyme and consonant frame nonwords. Fallon et al. hypothesized that the final biphone in rhyme words serves as a category cue in item recall. The consonant frame too may similarly serve as a category cue, so one may expect item recall of the two categories to be superior to both distinct and similar nonrhyme lists.

A characteristic of the serial recall task is that list items are more or less unrelated to one another and are presented in a random order. It is therefore unlikely that a participant has encountered the items previously in exactly the same order, even if all the items are familiar. Thus, per- 
formance in the ISR task will be influenced by both item and order memory. However, each category of scores in this task (i.e., serial recall, item recall, and position accuracy) and error scores such as intrusions and transpositions will all be affected by both item and order memory. Saint-Aubin and Poirier (2000) have argued, item and order errors are not empirically independent. The probability of an order error will increase with the number of items recalled. They suggested that order or transposition errors should therefore be measured as proportion of order errors per item recalled. In accord with this condition, we shall look for any indications in the pattern of recorded errors of whether order memory is affected differently by the four phonological categories, and we will test whether a reversal of PSE for nonword trigrams is associated with an advantage of item recall.

Some studies have shown that PSE is associated mainly with confusion of serial order or transpositions among the phonologically similar items in the list (Conrad, 1965; Drewnowski, 1980; Ellis, 1980; Healy, 1974; Murdock \& vom Saal, 1967). Item errors-such as omissions, repetitions, missed repetitions, and intrusions - do not increase to the same extent. Lian et al. (2004) found that order errors were more frequent in the recall of phonologically similar lists (e.g., rhyme and consonant frame lists), as compared with phonologically distinct lists both for words and nonwords, but across conditions transposition errors dominated in the serial recall of words and item errors dominated in the serial recall of nonwords. Although we are not in a position to infer from the error category the specific type of information lost in recall (Neath, 1999), we believe this finding indicates that phonological similarity is more detrimental to order memory of words than of nonwords, whereas other factors such as category cues or interlist similarities contribute to the advantages of phonological similarities in the recall of nonwords.

Despite the reversal of PSE for nonwords, as measured by serial recall and item score (Lian et al., 2004; Lian et al., 2001), phonological similarity may still have a detrimental effect on position accuracy for both words and nonwords (Lian et al., 2004). One may question whether phonological similarity is generally detrimental to order memory. In that case, phonologically similar lists such as rhyme and consonant frame lists should be inferior to distinct lists in the serial recognition task. Lian et al. (2001) found no effect of phonologicalsimilarity when serial recognition of similar nonrhyme trigrams was compared with serial recognition of distinct trigrams. However, rhyme and consonant frame lists consist of nonwords that share two out of three constituent phonemes, whereas similar nonrhyme nonwords share only one phoneme. Thus, in Experiment 2 we compared serial recognition of rhyme and consonant frame lists with serial recognition of distinct lists under conditions in which phonological similarity was aggravated by including two shared phonemes in the former list categories. By referring to rhyme, consonant frame, similar nonrhyme, and distinct lists in the present study, we distinguish between phonological simi- larities at the item level. It is important to note, however, that one may also distinguish between similarities at the list level, particularly when conditions are presented in a block-wise manner. This second type of similarity is likely to affect memory performance (see Lian et al., 2004), but is not the topic of the present study.

\section{EXPERIMENT 1}

The present experiment differed from Lian et al.'s (2004) Experiment 1 in two ways. First, it made use of a similar nonrhyme condition - that is, lists of items that share a vowel (e.g., fab, laf, sav). Second, repetitions of items did not occur. New items were presented in every trial. This was done to reduce sources of cross-trial interference for all phonological categories. In Lian et al.'s (2004) Experiment 1, recall of distinct lists, which were created by combining items from phonologically similar lists, was relatively more burdened with this type of interference than was recall of the phonologically similar items, because lists within the blocks of phonologically distinct trials were less distinguishable from one another than were lists within the blocks of phonologically similar trials. For this reason, Lian et al. (2004) suggested that a specification of stimulus materials should be made at both list and item levels. In other words, recall of indistinct lists of distinct items was compared with the recall of distinct lists of indistinct items. In the present experiment, cross-trial interference was reduced by making conditions more similar at the list level while phonological differences were maintained at the item level.

\section{Method}

Participants. Thirty-two psychology students from the University of Oslo (13 men and 19 women; age range, 18-36 years) volunteered to participate in the experiment. All reported Norwegian as their first language and reported no documented hearing or language disability.

Materials. To optimize comparisons between the categories of phonological similarity with respect to the recall of both item and order information, we tried to minimize the effects of interlist interference by using different items on every trial. PSE will occur also when no items are repeated between trials (Coltheart, 1993) and when testing is done immediately after list presentation (Nairne \& Kelley, 1999). A total of 96 CVC trigrams were used in the experiment. They were selected on the basis of two criteria. (1) The trigrams had no, or very few, single form occurrences in The Oslo Corpus of Tagged Norwegian Texts (2000), and (2) they could be sorted into one of four categories of phonological similarity (rhyme, consonant frame, distinct, or similar nonrhyme), with 24 items in each category. Four lists of six items were created within each phonological category. All trigrams were read with a short vowel by a speechtrained female actor and digitized at a $44.1 \mathrm{kHz}$ rate. A short vowel is generally represented by a following double consonant (e.g., $k k$ or $t t$ ) in written Norwegian. Hence, we recorded the number of singleform occurrences of the double-end consonant (CVCC) items in The Oslo Corpus (see Columns 4 and 8 of the Appendix). Ninety-one percent of the CVCC forms of the selected trigrams had zero occurrences in The Oslo Corpus.

The four categories of study lists were as follows: (1) lists of rhyming items that formed consonant contrasts - that is, the trigrams varied only in the initial consonant (e.g., tiv div kiv piv riv 
liv) - which shall henceforth be referred to as the rhyme category of items; (2) lists of rhyming items that formed vowel contrasts - that is, they varied only in their middle vowel (e.g., kab keb kob kåb kib $k u b$ ) — which shall be referred to as the consonant frame category of items; (3) lists of phonologically distinct items (e.g., vur nev kon sid båp $\mathrm{kad}$ ), which shall be referred to as the distinct category of items; and (4) lists of items that shared a vowel sound but differed with respect to initial and final consonant sounds (e.g., fab laf sav), which shall be referred to as the similar nonrhyme category of items.

The trigrams were selected with due consideration to phonological syntax and phonotactic frequencies in Norwegian. The phonotactic frequencies in the spoken language could not be assessed directly but were estimated indirectly from biphone frequencies in The Oslo Corpus (2000). We recorded the number of word forms with $\mathrm{CV}$ as the initial biphone and also recorded the number of word forms with $\mathrm{VC}$ as the final biphone. The sum of the two frequencies, $\mathrm{CV}+\mathrm{VC}$, was assumed to be closely related to the phonotactic frequency of the trigram in spoken Norwegian. By transforming the sums into natural logarithms, we were able to compare mean phonotactic frequencies for the four categories of lists. Paired $t$ tests of independent samples showed no significant differences between any of these categories.

Procedure. The sequence of lists within a phonological category remained constant, and the sequence of categories was balanced according to a Latin square design. The lists were presented via two loudspeakers, and particular sounds signaled the start and end of each list. The participants were instructed to recall the lists by saying aloud each of the nonwords in the correct order. In positions in which no item could be recalled, the participants were instructed to say "blank" or "no." The serial recall tasks were exemplified by way of two lists that were presented for practice. The participants were randomly assigned to one of two groups that differed with respect to the rate of the presentation of items. In one group, the nonword items were presented with an interstimulus interval (ISI) of $100 \mathrm{msec}$, and in the other group, the items were presented with an ISI of $500 \mathrm{msec}$.

The experimenter recorded all the responses by hand, and an audiotaped version of the responses was inspected to control on-line scoring. The experimenter scored correct-in-position items, missing items, transpositions, and item errors. The latter category was split into phonologically inaccurate responses and intrusions from other lists. Transpositions were recorded both per item presented and per item recalled. The error categories were defined according to traditional distinctions in the research literature (Ellis, 1980; Gathercole et al., 2001; Saint-Aubin \& Poirier, 2000) and do not imply a specification of the information lost in recall.

A strict scoring regime was maintained - that is, a response was scored as correct only when all three phonemes of the trigram were correctly repeated. The participants signed an informed consent and were debriefed by way of a short note regarding the purpose of the experiment.

Design. The experiment was run according to a $(2 \times 4)$ mixed design, with ISI as a between-groups variable of two levels and phonological similarity as a within-participants variable of four levels.

\section{Results}

Fallon et al.'s (1999) three scores of recall were applied: The serial recall score is the number of items recalled in correct position, item recall is the number of items recalled regardless of position, and position accuracy is the serial recall to item recall ratio. A repeated measures analysis of variance (ANOVA) with one between-groups variable showed no main effect of presentation rate for any of the scoring procedures. A main effect of phonological similarity was observed for all three scores [serial recall, $F(3,90)=25.12, p<.001 ;$ item recall, $F(3,90)=131.08$, $p<.001$; position accuracy, $F(3,90)=5.11, p=.003]$. There was no significant interaction between presentation rate and phonological similarity for any of the scoring procedures, and we therefore collapsed the data across presentation rate in the following analyses. Table 1 shows the mean proportions of serial recall, item recall, and position accuracy according to phonological similarity for the entire data set.

Post hoc repeated measures $t$ tests with sequential Bonferroni adjustments $(\alpha=.05)$ were undertaken for the three recall scores. We found that serial recall was significantly higher for consonant frame items than for rhyme $[t(31)=4.68, p<.05]$, similar nonrhyme $[t(31)=6.16$, $p<.05]$, and distinct $[t(31)=6.91, p<.05]$ items. Rhyme lists did not differ significantly from similar nonrhyme and distinct lists.

Bonferroni-adjusted post hoc comparisons revealed that item recall of consonant frame lists was significantly higher than that of rhyme $[t(31)=5.83, p<.05]$, similar nonrhyme $[t(31)=15.99, p<.05]$, and distinct $[t(31)=$ $14.40, p<.05]$ lists. Also, item recall of rhyme lists was significantly higher than item recall of both similar nonrhyme $[t(31)=11.16, p<.05]$ and distinct $[t(31)=11.50$, $p<.05]$ lists.

The same post hoc comparisons revealed that position accuracy was better for consonant frame than for rhyme lists $[t(31)=2.91, p<.05]$. Similar nonrhyme lists were recalled more accurately than were rhyme lists $[t(31)=$ $4.75, p<.05]$, and distinct lists were recalled more accurately than were rhyme lists $[t(31)=2.89, p<.05]$. Comparisons were nonsignificant between consonant frame, similar nonrhyme, and distinct lists.

Error analysis. We adopted the traditional distinction between omissions, item errors, and order errors. The former type included all cases of blank responses. Item errors were split into two subcategories: intrusions from other lists and phonologically inaccurate items that were not whole-item intrusions from other lists. Order errors were items from the original list that were recalled in an incorrect position, including items that were repeated in an incorrect position. This category was split into proportions of order errors per item presented and proportions of order errors per item recalled. The protocol of error scores is exhaustive in the sense that the sum of omissions, item errors, and order errors (per item presented) equals 1 minus the serial recall score. Table 2 shows the proportions of errors in five error categories for the four phonological cat-

\section{Table 1}

Mean Proportions and Standard Deviations of Correct Recall for Rhyme, Consonant Frame, Similar Nonrhyme, and Distinct Trials With Three Scoring Procedures in Experiment 1

\begin{tabular}{|c|c|c|c|c|c|c|c|c|}
\hline \multirow[b]{2}{*}{ Score } & \multicolumn{2}{|c|}{ Rhyme } & \multicolumn{2}{|c|}{$\begin{array}{c}\text { Consonant } \\
\text { Frame }\end{array}$} & \multicolumn{2}{|c|}{$\begin{array}{c}\text { Similar } \\
\text { Nonrhyme }\end{array}$} & \multicolumn{2}{|c|}{ Distinct } \\
\hline & $M$ & $S D$ & $M$ & $S D$ & $M$ & $S D$ & $M$ & $S D$ \\
\hline Serial recall & .25 & .16 & .41 & .19 & .19 & .11 & .18 & .11 \\
\hline Item recall & .58 & .15 & .73 & .16 & .31 & .10 & .31 & .12 \\
\hline Recall accuracy & .42 & .26 & .55 & .21 & .63 & .29 & .62 & .37 \\
\hline
\end{tabular}


Table 2

Proportions of Errors in Experiment 1 According to Error Category and Mean Proportions of Errors Across List Categories

\begin{tabular}{lcccccc}
\hline \multirow{2}{*}{ List } & \multicolumn{2}{c}{ Item Errors } & & \multicolumn{2}{c}{ Order Errors Per Item } & \\
\cline { 2 - 3 } & Phon & Intr & & Presented & Recalled & Omissions \\
\hline Rhyme & .10 & .02 & & .33 & .58 & .30 \\
Consonant frame & .04 & .01 & .32 & .45 & .22 \\
Distinct & .33 & .03 & .13 & .42 & .33 \\
Similar nonrhyme & .32 & .02 & .11 & .37 & .36 \\
$M$ & .20 & .02 & .22 & .46 & .30 \\
\hline
\end{tabular}

Note-Phon, phonologically inaccurate responses; Intr, intrusions from other lists.

egories. A 5 (error categories) $\times 4$ (phonological categories) repeated measures ANOVA showed a main effect of error type $[F(4,124)=73.44, p<.001]$ that was mainly caused by the low frequency of intrusions from other lists. A main effect of phonological similarity $[F(3,93)=5.25$, $p=.002]$ showed that errors were relatively rare in the recall of consonant frame and rhyme lists, as compared with the other list categories. A significant interaction between error type and phonological similarity $[F(12,372)=24.05$, $p<.001]$ showed that item errors accrued to distinct and similar nonrhyme lists and were particularly rare in the recall of consonant frame lists. Conversely, order errors were more frequent in the rhyme and consonant frame lists.

Paired $t$ tests for differences between mean error scores in the four phonological categories were undertaken with Bonferroni corrections. The four categories yielded six comparisons, and with a family-wise error rate of .05 , the critical $t=2.635$. The tests showed that error frequency for rhyme lists differed significantly from that for consonant frame lists $[t(31)=4.68, p<.05]$ but not from error frequency for distinct and similar nonrhyme lists. Moreover, error frequency for consonant frame lists differed significantly from that for both distinct $[t(31)=6.16, p<$ $.05]$ and similar nonrhyme $[t(31)=6.91, p<.05]$ lists.

Intrusions of whole items from other lists formed an inconsiderable part of item errors. Yet such errors were significantly more frequent for distinct lists than for consonant frame $[t(31)=4.69, p<.05]$ and similar nonrhyme $[t(31)=3.11, p<.05]$ lists, but not for rhyme lists. Item errors that constituted phonologically inaccurate responses were significantly more frequent in the distinct category than in both consonant frame $[t(31)=17.68$, $p<.05]$ and rhyme $[t(31)=12.31, p<.05]$ lists. Also, this type of item error was significantly more frequent for similar nonrhyme lists than for rhyme lists.

We undertook $t$ tests for differences of order errors between phonological categories for order errors computed both per item presented and per item recalled. The results of these tests are summarized in Table 3. The significant differences were between the rhyme and distinct categories and between the rhyme and similar nonrhyme categories, a result that demonstrates the domination of order errors for rhyme lists in terms of order errors both per item presented and per item recalled. The consonant frame category differed significantly from both distinct and similar nonrhyme categories in respect to order errors per item presented but not in respect to order errors per item recalled. In contrast, rhyme and consonant frame lists, which did not differ with respect to order errors per item presented, did differ with respect to order errors per item recalled.

We also undertook $t$ tests for differences of mean omissions with Bonferroni corrections. These tests showed that consonant frame lists gave rise to significantly fewer omissions than did both distinct $[t(31)=3.20, p<.05]$ and similar nonrhyme $[t(31)=4.56, p<.05]$ lists.

\section{Discussion}

The present experiment investigated whether Fallon et al.'s (1999) findings for words extend to nonwords. Effects of phonological similarity in the two experiments can be compared only by considering the recall data for rhyme, similar nonrhyme, and distinct lists. The fourth category, consonant frame lists, represents a new operationalization of phonological similarity. A specific discussion of the recall data for this category will be undertaken later.

Fallon et al.'s (1999) study confirmed the classical PSE for words when recall of similar nonrhyme words was compared with recall of distinct words, in terms of both serial recall and item recall scores. The present study shows that PSE was absent for nonwords when recall scores of the same item categories were compared. That is, there were no significant differences in serial recall and item recall for these categories of nonwords. The absence of PSE as measured by serial recall for distinct and similar nonrhyme lists seems consistent with Lian et al.'s (2001) study. They found a reversal of PSE for nonwords of low associative value when the same item categories were compared on the serial recall score. The nonword trigrams in the present experiment were selected on the basis of frequency counts in The Oslo Corpus (2000) without regard to associative value. Therefore, the present results may be a combined effect of nonword items of relatively high associative value that favor the recall of distinct items and nonword items of low associative value that favor the recall of similar nonrhyme items.

The contrasting results between Fallon et al.'s (1999) and the present study with respect to distinct and similar nonrhyme items, in terms of both serial and item recall,

\section{Table 3}

Results of $t$ Tests of Differences of Means in Order Error Scores With Bonferroni Corrections for Six Paired Comparisons

\begin{tabular}{|c|c|c|c|c|c|c|}
\hline \multirow[b]{2}{*}{ Comparisons } & \multicolumn{3}{|c|}{$\begin{array}{c}\text { Per Item } \\
\text { Presented }\end{array}$} & \multicolumn{3}{|c|}{$\begin{array}{l}\text { Per Item } \\
\text { Recalled }\end{array}$} \\
\hline & $t$ & $d f$ & $p$ & $t$ & $d f$ & $p$ \\
\hline Rhyme, consonant frame & .12 & 31 & .05 & 2.91 & 31 & $<.05$ \\
\hline Rhyme, distinct & 8.29 & 31 & $<.05$ & 2.80 & 31 & $<.05$ \\
\hline Rhyme, similar & 8.65 & 31 & $<.05$ & 4.75 & 31 & $<.05$ \\
\hline Consonant frame, distinct & 6.57 & 31 & $<.05$ & .39 & 31 & $>.05$ \\
\hline Consonant frame, similar & 7.57 & 31 & $<.05$ & 1.59 & 31 & $>.05$ \\
\hline Distinct, similar & .88 & 31 & $>.05$ & 1.14 & 31 & $>.05$ \\
\hline
\end{tabular}

Note-Critical $t=2.635$, with desired family-wise error rate $=.05$. 
are consistent with Lian et al.'s (2001) arguments that different long-term memory mechanisms are involved in serial recall of words and nonwords. However, the results from the two studies do not contrast insofar as the recall data for distinct and rhyme lists are concerned. We found a reversal of PSE in terms of both serial and item recall. Fallon et al. demonstrated a reversal for item recall and an absence of PSE for serial recall. Both studies showed relatively good recall of rhyme items, particularly when measured as item recall, as compared with distinct and similar nonrhyme items. This result indicates a possible category cuing effect that occurs for both words and nonwords.

The present results do not provide much support for the hypothesis that position accuracy is an inverse function of the number of shared phonemes in the presented list. Both rhyme and consonant frame lists were recalled with less accuracy than were similar nonrhyme and distinct lists. But position accuracy for consonant frame lists did not differ significantly from that for similar nonrhyme and distinct lists. Nor did the latter categories differ with respect to position accuracy. Yet the results are not entirely inconsistent with the hypothesis.

Why is recall of items from rhyme lists less accurate than recall of items from consonant frame lists? The recall data for rhyme lists generally agrees with the results reported by Fallon et al. (1999). These researchers attributed the superior item scores for rhyme items (i.e., consonantcontrasting items such as cat and rat) to a category cuing effect, but they claimed that "the advantage that category membership produces with item information disappears with position accuracy scoring" (Fallon et al., 1999, p. 303). In the present experiment, however, the item recall advantage caused by a category cuing effect disappeared with position accuracy scoring for rhyme but not for consonant frame items, although both categories included phonemic segments that were likely to serve as category cues. Lian et al. (2004) demonstrated that the position accuracy advantage for consonant frame lists, relative to rhyme lists, applies to both words and nonwords. The present study replicated this advantage of consonant frame trigrams when an open set was used, showing that the position accuracy difference between two types of lists is a robust effect. If a cue is loaded with targets (see Nairne, 1990) — as should be the case if the shared phonemes are employed as a retrieval cue-then item recall should increase and position accuracy should decrease. In the present study, this pattern differed somewhat for rhyme and consonant frame lists. Consonant frame lists had better item recall scores and position accuracy than did rhyme lists. It seems as if vowel variation causes greater distinctiveness than consonant variation does (see Surprenant \& Neath, 1996), but it is not entirely clear why this is so. In any case, vowel variation is associated with higher position accuracy than consonant variation is. It may be that consonant frame lists have both the cuing advantage of rhyme lists and the phonological distinctiveness advantage of distinct lists.
The recall data for consonant frame items in the present study give rise to particular questions that are rarely discussed in the experimental literature on phonological short-term memory. Why are consonant frame nonwords recalled better than nonwords are in the rhyme category? Item memory may depend on the size of the category, which may explain the difference in item scores between consonant frame and rhyme nonwords. The lists of consonant frame nonwords represent series of vowels, whereas the rhyme lists represent series of consonants. Whether the size of these categories also explains the recency advantage for vowels, as compared with consonants (Crowder, 1971), is hard to say. But the fact that there are more consonants than vowels in most languages cannot explain why the serial position of items with differing vowels is recalled more accurately than is the serial position of items with differing consonants. Surprenant and Neath (1996) made use of a serial reconstruction task and found that memory performance for vowel-contrasting syllables was better than that for consonant-contrasting syllables, even when vowels were made less discriminable than consonants were. This result agrees with the present finding that position accuracy of vowels is better than position accuracy of consonants. Also, the present recall data for consonant frame nonwords, as measured with all three scoring criteria, agrees with the recall data for consonant frame words reported in Lian et al.'s (2004) study.

Evidence of superior performance for consonant frame lists in the serial recall task also appeared in the error analysis. Recall of items from this category of lists was less burdened with errors across all error categories than was recall of the other list categories. In particular, we found item errors to be highly infrequent with consonant frame lists. However, the distribution of item and order errors according to phonological categories does not imply a specification of the information lost in recall. Thus, Neath (1999), in a simulation of a serial reconstruction experiment, argued that experimental manipulations affecting item information may produce order errors and vice versa. Yet the distribution of item and order errors in the present ISR task may nonetheless be related to different sources of interference. Therefore, the distribution may provide some indication of what information is lost in recall. The consonant frame category is constituted of highly distinct lists within the total set of study items, and yet these lists include phonologically similar or indistinct items. This property of consonant frame lists reduces the likelihood of interlist interference and, therefore, of intrusions of items from other lists. The distinctiveness of rhyme lists works similarly to lower the frequency of intrusions from other lists, although not to the extent that is found for consonant frame lists. In general, intrusions from other lists were rare, owing to the open set of study items. Other item errors were phonologically inaccurate responses that accrued mainly to distinct and similar nonrhyme lists. In contrast to the distinctiveness of the items in the rhyme and consonant frame lists, there was a high degree of similarity among items within these two categories that in- 
creased the likelihood of intralist interference and that consequently gave rise to more order errors. Order errors per item recalled accrued more to rhyme lists than to any of the other list categories. Thus, it seems likely that memory of order is disadvantaged for items in rhyme lists, as compared with other list categories, including the consonant frame lists. Also, rhyme lists were recalled with less position accuracy than were the other list categories. This raises the question of whether the difference of position accuracy between rhyme and consonant frame items reflects a general advantage for vowels in tasks that require memory of order. In that case, we would expect better memory performance for vowels than for consonants in a serial recognition task (Allport, 1984; Gathercole et al., 2001; Martin, Lesch, \& Bartha, 1999). This problem was studied in the next experiment.

\section{EXPERIMENT 2}

Serial recognition tasks are in certain ways less demanding than ISR tasks are. First, the presentation of test items works as an effective retrieval cue, and second, since the serial recognition task involves the use of yes/no decisions, inconsiderable strain is laid on the response system. The effect of phonological similarity on recognition performance may therefore differ from that on recall performance in the ISR task.

Gathercole et al. (2001) found a PSE for both words and nonwords in a serial recognition task for children aged 8-9 years, whereas Lian et al. (2001) demonstrated PSE for words but not nonwords of low associative value in a serial recognition task for adult participants. Differences in associative value and wordlikeness of the nonwords used in the two studies may account for the divergent results on nonword serial recognition. The nonwords in Lian et al.'s (2001) study were CVC trigrams sorted into distinct and similar nonrhyme lists. Lists of distinct nonwords always differed with respect to the medial vowel and included no more than two items with the same initial or final consonant. Lists of similar nonwords always shared the medial vowel. This category corresponded to the similar nonrhyme items in the present Experiment 1.

The present Experiment 2 will extend Lian et al.'s (2001) Experiment 2 by comparing serial recognition of distinct nonwords with the serial recognition of consonant frame and rhyme nonwords. In Experiment 1, the latter two categories differed significantly with respect to position accuracy - that is, consonant frame nonwords were recalled more accurately with respect to position than rhyme nonwords were. This result indicates better order memory for consonant frame nonwords. Does this result mean that serial recognition of consonant frame items is superior as compared with rhyme items but comparable with the serial recognition of distinct items?

\section{Method}

Participants. Thirty students from the University of Oslo (12 males and 18 females, age range 19-29 years) served as participants in the experiment. All the participants were native speakers of Nor- wegian, had no documented hearing or language disorder, and signed an informed consent before taking part in the experiment.

Materials. A subsample of $44 \mathrm{CVC}$ trigrams was selected from the set of nonwords used in Lian et al. (2004) on the basis of frequency counts in The Oslo Corpus (2000). As in Experiment 1, all the trigrams were read by a speech-trained female actor and were digitized at a $44.1 \mathrm{kHz}$ rate. Forty-five percent of the selected trigrams overlapped with the trigram set used in Experiment 1 . All the trigrams were nonwords in the lexical sense-that is, none of them constituted Norwegian words, although some of them may have sounded similar to Norwegian or foreign words. They were sorted into one of three categories varying in phonological similarity(1) rhyme items, (2) consonant frame items, and (3) phonologically distinct items - that were all defined in the same way as the item categories of Experiment 1 were. In the present experiment there was some overlap of items between the phonological categories, but the number of repeated items was balanced between categories. A minimum number of repetitions was necessitated by the serial recognition task. Each successively presented list pair contained the same items in the same or different order (e.g., biv bov bav buv-bov biv bav $b u v$ ). Each list pair was presented once in the same order and once in a different order, producing four repetitions of each item. Within each category, we constructed four lists of four items and four lists of five items, and each list gave rise to two pairs to be compared for serial order. Thus, within the category of consonant frame items at List Length 4, eight pairs of lists were presented, half of which included items in an identical order and the other half in a different order. The same number of pairs was presented for List Length 5 in each of the other phonological categories. In total, we had 216 single item presentations, which means that on the average each item was repeated five times. Thus, in addition to being repeated four times within a category, each item was also repeated once in another category.

Procedure. The participants were presented with a pair of lists separated by a 2 -sec pause, and interitem pauses within a list were $500 \mathrm{msec}$. The participants were told that the two lists included exactly the same items, sometimes in a different order with only two items transposed into adjacent positions in the second presentation (i.e., a minimal change of order) and sometimes in exactly the same order. Their task was to make a yes/no decision with respect to similarity of order.

The sequence of phonological categories was balanced according to a Latin square design, but within each category, List Length 4 always preceded List Length 5 . Identical and different pairs of lists were randomized within each phonological and list length category.

Design. The experiment was run according to a repeated measures design with the factors list length (2) and phonological category (3).

\section{Results and Discussion}

Table 4 presents the mean proportion of yes/no decisions as a function of list length and phonological category and across list lengths for each category. A repeated measures ANOVA revealed a main effect of phonological similarity $[F(2,58)=3.68, p=.031]$ but no significant main effect of list length. There was a significant interaction between list length and phonological similarity $[F(2,58)=12.63, p<.001]$, since the effect of phonological similarity is stronger at List Length 4 . At List Length 5 the task becomes considerably more difficult, and an element of guessing cannot be excluded since the probability of making six correct yes/no decisions out of eight by chance is .10. For List Length 4 , paired $t$ tests with Bonferroni adjustments and three post hoc comparisons ( $\alpha=.05$ ) showed that serial recognition of consonant 
Table 4

Mean Proportions and Standard Deviations of Correct Yes/No Decisions on a Serial Recognition Task in Experiment 2

\begin{tabular}{|c|c|c|c|c|c|c|}
\hline \multirow[b]{2}{*}{ List } & \multicolumn{2}{|c|}{ Rhyme Items } & \multicolumn{2}{|c|}{ Consonant Frame Items } & \multicolumn{2}{|c|}{ Distinct Items } \\
\hline & $M$ & $\overline{S D}$ & $M$ & $S D$ & $M$ & $S D$ \\
\hline 4 & .69 & .19 & .84 & .17 & .74 & .20 \\
\hline 5 & .62 & .17 & .65 & .20 & .63 & .16 \\
\hline$M$ & .66 & .15 & .75 & .14 & .69 & .15 \\
\hline
\end{tabular}

frame lists was significantly better than that for both distinct $[t(29)=2.63, p<.05]$ and rhyme $[t(29)=3.89, p<$ $.05]$ lists. No significant difference was revealed between rhyme and distinct lists. When differences between mean proportions across list lengths were tested, we found that memory performance for consonant frame lists was significantly better than that for list pairs in the rhyme category $[t(29)=2.65, p<.05]$. The difference of means between serial recognition of distinct and consonant frame lists yielded a result $[t(31)=2.39]$ that was close to but did not exceed the Bonferroni correction $(t=2.3954$ for three tests and an error rate of .05).

The results demonstrate that serial recognition of consonant frame lists is superior to serial recognition of rhyme lists. The order of consonant frame nonwords is recognized better than the order of distinct nonwords at List Length 4, but not across the entire data set. The fact that serial recognition of consonant frame lists was not superior to that of distinct nonwords across list lengths may have been caused by the variation of medial vowels in the distinct lists. Both the consonant frame and distinct lists included vowel differences. Therefore, vowel variation is likely to be a critical factor that facilitates the memory of order.

\section{GENERAL DISCUSSION}

The present study yielded evidence of two opposing effects of phonological similarity in serial recall of nonwords. In agreement with Lian et al. (2004), PSE was reversed in both the classical serial recall score and the item recall score when distinct lists were compared with rhyme and consonant frame lists. Yet PSE was present when position accuracy scores of distinct and rhyme lists were compared. PSE was absent for all scoring criteria when similar nonrhyme and distinct lists were compared.

The results of the present study agree with Fallon et al.'s (1999) results for rhyme lists with the item recall and position accuracy criteria. Thus, words and nonwords are equally affected by the rhyme operationalization of phonological similarity when performance is assessed in terms of item recall and position accuracy. When performance is assessed in terms of serial recall, however, effects of all three forms of phonological similarity differ between words and nonwords.

PSE in serial recognition of nonwords is reversed when distinct and consonant frame lists of List Length 4 are compared and are absent when distinct lists are compared with both rhyme and consonant frame lists across list lengths. Yet consonant frame lists have an advantage in se- rial recognition tasks, since the serial order of these lists is recognized significantly better than the serial order of rhyme lists. At the same time, the difference between consonant frame and distinct lists is close to significance with the Bonferroni correction, but this result constitutes no convincing evidence of a reversal of PSE in the serial recognition task for nonwords.

The effects of phonological similarity clearly depend on (1) the way we define phonological similarity and (2) the way we score recall performance in the serial recall task. The results of Experiment 1 contrast with those of similar experiments that used words in serial recall tasks (Baddeley, 1968; Coltheart, 1993), indicating that detrimental effects of phonological similarity are limited to memory of word sequences. However, the position accuracy scores in Experiment 1 do not fully support this conclusion. In this experiment, and in Fallon et al.'s (1999) Experiments 1 and 2 , rhyme lists were recalled less accurately than were phonologicallydistinct lists. These findings support a tentative conclusion that the detrimental effects of phonological similarity are more evident in position accuracy scores than in serial recall scores in serial recall tasks. Furthermore, they support the general assumption that similarity, be it phonological or semantic, is mainly a disadvantage to order memory (Conrad, 1965; Drewnowski, 1980; SaintAubin \& Poirier, 2000).

Fallon et al. (1999) showed that rhyming words (with consonant contrasts) and similar nonrhyming words were recalled less accurately than phonologicallydistinct words were. This finding has given rise to the hypothesis that position accuracy is an inverse function of the number of shared phonemes of the items in the presented list. A confirmation of that hypothesis would support the assumption that phonological similarity is detrimental to order memory. However, the present study has given only partial support to this hypothesis. Consonant frame and rhyme lists, in which items of both categories share two phonemes, differed significantly with respect to position accuracy, and lists of similar nonrhyming nonwords, which share one phoneme, did not differ from lists of distinct nonwords, which have no phoneme in common.

Fallon et al.'s (1999) interpretation, that of the serial recall scores for rhyme lists as the masking of a positive effect of category cuing in item recall with the detrimental effect of phonological similarity in position accuracy, is not supported by the present data. Lists of both rhyme and consonant frame nonwords include shared phonemic elements, and therefore, category cuing should be effective in the recall of both types of lists. Yet item recall of consonant frame lists was significantly better than item recall of rhyme lists.

Both categories excelled in relation to similar nonrhyme and distinct lists, with respect to both serial recall and item recall. Note that both rhyme and consonant frame lists differ substantially from similar nonrhyme lists in phonological structure, since items in each of the former two categories share two phonemes whereas items in the latter category share only one phoneme. Yet only rhyme items were disadvantaged with respect to position accu- 
racy in the serial recall task. The position accuracy of consonant frame items differed significantly from neither distinct nor similar nonrhyme lists.

In view of both the present study and that of Fallon et al. (1999), it may be argued that similarity, be it phonological or semantic, is an impediment to order memory. However, a detrimental effect of phonological similarity may be restricted to order memory for words, or else, in view of the present results, the effect applies only to a certain operationalization of phonological similarity.

Note that memory of consonant frame items is better than memory of rhyme items, in terms of all three recall scores in the serial recall task of Experiment 1 and in terms of serial recognition in Experiment 2. This result indicates that both position accuracy and serial recognition represent memory of order and that vowels (in consonant frame lists) facilitate memory of order more than do consonants (in rhyme lists). The present findings, therefore, point to the roles of consonant and vowel sounds in shortterm memory and should be discussed in relation to the research tradition starting with the works of Crowder (1971), Crowder and Morton (1969), and Pisoni (1973, 1975). These investigators focused on vowel and consonant sounds in relation to such specific effects as recency effects, suffix effects, and modality effects, as well as the relative discriminability of the two speech categories. The differences between vowel and consonant sounds with respect to categorical perception were discussed by Pisoni (1973), whereas Surprenant and Neath (1996) discussed the relation between discriminability and memory of speech sounds.

We assume that both the consonant frame $\left(\mathrm{C}_{-} \mathrm{C}\right)$ and the final biphone (VC) of rhyme lists may serve as retrieval cues in the serial recall task. The present data show that C_C may be a more effective cue than is the rhyme element VC. However, these cues are also associated with different series of phonemic contrasts. Thus, the consonant frame C_C is associated with series of vowel contrasts, and the rhyme element $\mathrm{VC}$ is associated with series of consonant contrasts. Therefore, we cannot tell whether the advantage of consonant frame lists, as compared with that of rhyme lists, can be attributed to the invariant cue or the phonemic contrasts. Yet vowel variation alone (in distinct lists) improves position accuracy for both words (Fallon et al., 1999) and nonwords (Lian et al., 2004). Vowel variation also improves, although to a lesser degree, position accuracy for consonant frame lists. Also, order errors per item recalled are less frequent for consonant frame than for rhyme lists. Thus, position accuracy, together with order errors per item recalled, show that vowel variation facilitates short-term memory of order. The advantage of vowel variation over consonant variation has previously been shown in a task of serial reconstruction (Surprenant \& Neath, 1996). Further support to this conclusion is given by the results of Experiment 2, which show an advantage for the consonant frame over rhyme lists in the serial recognition task.

In view of the present study, a readjustment of Gathercole and Martin's (1996) interactive model is needed. We have suggested that PSE for words is associated with an inhibition of representations of phonological neighbors in the primary lexical-phonologicallayer. However, we have not so far introduced a precise definition of phonological neighborhood. The present study, Fallon et al. (1999), and Lian et al. (2004) have all indicated that vowel variation creates phonological contrasts rather than phonological neighbors - that is, consonant frame items will not be represented as phonological neighbors. Items that share a vowel sound are more likely to be represented as phonological neighbors the more consonant sounds they share. Furthermore, the inhibition of such neighbors is detrimental to their serial order in memory tasks. Finally, this inhibition is limited to representations in the primary lexicalphonological layer (i.e., representations of words and high associative nonwords).

The recall of nonwords that are represented as new, unstable clusters in a prelexical phonological layer should not be affected by phonological similarity. In the present study, only nonwords that formed rhyme lists were thus affected, and only when memory performance was measured in terms of position accuracy. This is not surprising, since it may be the case that the rhyme category was a mixture of items of low and high associative values whose representations are close phonological neighbors.

Shared phonemes are an effective category cue during retrieval of rhyme lists and consonant frame lists, regardless of lexicality and associative value of the study items. Shared phonemes may also be used as a category cue during retrieval of similar nonrhyme lists. However, it would not be as effective as in the other categories of phonological similarity because the final consonant is not a constant, which causes a greater proportion of item errors. Shared phonemes also function as a category cue in longterm memory, as is seen in the reversal of PSE in delayed recall of words (Lian et al., 2004). The advantage of phonologically distinct items over rhyme and consonant frame items is restricted to items that are perceived as sufficiently meaningful or familiar, and only in cases of immediate recall, not delayed recall. With phonologically similar nonwords that share the midvowel, however, the reversal of PSE is less consistent and is related to the associative value of the nonwords. The superiority of distinct lists appears to be a genuine effect of primary memory or an on-line language mechanism, whereas the superiority of rhyming relies on the organization of long-term memory in the construction of response alternatives. Paradoxically, the former effect applies only to semantically distinct material (i.e., material that readily actives longterm memory representations), whereas the latter effect may be applied to any verbal material.

\section{REFERENCES}

Allport, D. A. (1984). Auditory-verbal short-term memory and conduction aphasia. In H. Bouma \& D. G. Bouwhuis (Eds.), Attention and performance X: Control of language processes (pp. 313-326). London: Erlbaum.

BADDELEY, A. D. (1966). The influence of acoustic and semantic simi- 
larity on long-term memory for word sequences. Quarterly Journal of Experimental Psychology, 18, 302-309.

BADDELEY, A. D. (1968). How does acoustic similarity influence shortterm memory? Quarterly Journal of Experimental Psychology, 20, 249-263.

BADDEley, A. D. (1986). Working memory. Oxford: Oxford University Press.

BAdDeley, A. D. (2001). Is working memory still working? American Psychologist, 56, 851-864.

BARTLETt, F. C. (1932). Remembering: A study in experimental and social psychology. Cambridge: Cambridge University Press.

BESNER, D., \& DAvelaAR, E. (1982). Basic processes in reading: Two phonological codes. Canadian Journal of Psychology, 36, 701-711.

Brown, G. D. A., \& Hulme, C. (1995). Modelling item length effects in memory span: No rehearsal needed? Journal of Memory \& Language, 34, 594-621.

BURGESS, N., \& Hitch, G. J. (1992). Toward a network model of the articulatory loop. Journal of Memory \& Language, 31, 429-460.

Burgess, N., \& Hitch, G. J. (1999). Memory for serial order: A network model of the phonological loop and its timing. Psychological Review, 106, 551-581.

Coltheart, V. (1993). Effects of phonological similarity and concurrent irrelevant articulation on short-term-memory recall of repeated and novel word lists. Memory \& Cognition, 21, 539-545.

CONRAD, R. (1965). Order error in immediate recall of sequences. Journal of Verbal Learning, 4, 161-169.

CONRAD, R., \& HulL, A. J. (1964). Information, acoustic confusion and memory span. British Journal of Psychology, 55, 429-432.

CROWDER, R. G. (1971). The sound of vowels and consonants in immediate memory. Journal of Verbal Learning \& Verbal Behavior, 12, 599-607.

Crowder, R. G. (1979). Similarity and order in memory. In G. H. Bower (Ed.), The psychology of learning and motivation: Advances in research and theory (Vol. 13, pp. 319-353). New York: Academic Press.

Crowder, R. G., \& Morton, J. (1969). Precategorical acoustic storage (PAS). Perception \& Psychophysics, 5, 365-373.

DREWNOWSKI, A. (1980). Attributes and priorities in short-term recall: A new model of memory span. Journal of Experimental Psychology: General, 109, 208-250.

ELLIS, A. W. (1980). Errors in speech and short-term memory: The effects of phonemic similarity and syllable position. Journal of Verbal Learning \& Verbal Behavior, 19, 624-634.

Fallon, A. B., Groves, K., \& Tehan, G. (1999). Phonological similarity and trace degradation in the serial recall task: When CAT helps RAT, but not MAN. International Journal of Psychology, 34, 301-307.

GATHERCOLE, S. E. (1997). Models of verbal short-term memory. In M. A. Conway (Ed.), Cognitive models of memory (pp. 14-45). Cambridge, MA: MIT Press.

Gathercole, S. E., \& MARTin, A. (1996). Interactive processes in phonologicalmemory. In S. E. Gathercole (Ed.), Models of short-term memory (pp. 73-100). Hove, U.K.: Erlbaum.

Gathe rcole, S. E., Pickering, S. J., Hall, M., \& Peaker, S. M. (2001). Dissociable lexical and phonological influences on serial recognition and serial recall. Quarterly Journal of Experimental Psychology, 54A, 1-30.

HeALY, A. F. (1974). Separating item from order information in shortterm memory. Journal of Verbal Learning \& Verbal Behavior, 13, 644-655.

Hitch, G. J., \& HALlidAY, M. S. (1983). Working memory in children.
Philosophical Transactions of the Royal Society of London: Series B, 302, 324-340.

Hulme, C., Roodenrys, S., Brown, G. D. A., \& Mercer, R. (1995). The role of long-term memory mechanisms in memory span. British Journal of Psychology, 86, 527-536.

KARLSEN, P. J., \& LiAn, A. (2004). Modulating the phonological similarity effect: The contribution of interlist similarity and lexicality. Manuscript submitted for publication.

Lian, A., KARLSEn, P. J., \& ERIKSEn, T. (2004). Opposing effects of phonological similarity on item and order memory of words and nonwords in the serial recall task. Memory, 12, 314-337.

Lian, A., KARlsen, P. J., \& Winsvold, B. (2001). A re-evaluation of the phonological similarity effect in adults' short-term memory of words and nonwords. Memory, 9, 281-299.

Longoni, A. M., Richardson, J. T. E., \& Aiello, A. (1993). Articulatory rehearsal and phonological storage in working memory. Memory \& Cognition, 21, 11-22.

Martin, R. C., Lesch, M. F., \& Bartha, M. C. (1999). Independence of input and output phonology in word processing and short-term memory. Journal of Memory \& Language, 41, 3-29.

Murdock, B. B., \& VOM SAAL, W. (1967). Transpositions in short-term memory. Journal of Experimental Psychology, 74, 137-143.

NAIRne, J. S. (1990). A feature model of immediate memory. Memory \& Cognition, 18, 251-269.

NAIRNE, J. S., \& KELlEy, M. R. (1999). Reversing the phonological similarity effect. Memory \& Cognition, 27, 45-53.

NEATH, I. (1999). Modelling the disruptive effects of irrelevant speech on order information. International Journal of Psychology, 34, 410-418.

NeISSER, U. (1982). Snapshots or benchmarks? In U. Neisser (Ed.), Memory observed: Remembering in natural contexts (pp. 43-48). San Francisco: Freeman.

The Oslo corpus of tagged Norwegian texts (2000, June). Available at http://www.tekstlab.uio.no/norsk/bokmaal/english.html.

PAgE, M. P. A., \& NorRIs, D. (1998). The primacy model: A new model of immediate serial recall. Psychological Review, 105, 761-781.

PAPagno, C., \& VAllar, G. (1992). Phonological short-term memory and the learning of novel words: The effect of phonological similarity and item length. Quarterly Journal of Experimental Psychology, 44A, 47-67.

PISONI, D. B. (1973). Auditory and phonetic memory codes in the discrimination of consonants and vowels. Perception \& Psychophysics, 13, 253-260.

PISONI, D. B. (1975). Auditory short-term memory and vowel perception. Memory \& Cognition, 3, 7-18.

SAINT-Aubin, J., \& PoIRIER, M. (2000). Immediate serial recall of words and nonwords: Tests of the retrieval-based hypothesis. Psychonomic Bulletin \& Review, 7, 332-340.

SCHWEICKERT, R. (1993). A multinominal processing tree model for degradation and redintegration in immediate recall. Memory \& Cognition, 21, 168-175.

Schweickert, R., Guentert, L., \& Hersberger, L. (1990). Phonological similarity, pronunciation rate, and memory span. Psychological Science, 1, 74-77.

SurprenAnt, A. M., \& NeATh, I. (1996). The relation between discriminability and memory for vowels, consonants, and silent-center vowels. Memory \& Cognition, 24, 356-366.

WATKIns, M. J., WATKInS, O. C., \& CROWdeR, R. G. (1974). The modality effect in free and serial recall as a function of phonological similarity. Journal of Verbal Learning \& Verbal Behavior, 13, 430-447. 
APPENDIX

Trigram Counts in The Oslo Corpus

\begin{tabular}{|c|c|c|c|c|c|c|c|}
\hline Item & $\mathrm{CV}$ & $\mathrm{VC}$ & CVCC & Item & $\mathrm{CV}$ & $\mathrm{VC}$ & $\mathrm{CVCC}$ \\
\hline \multicolumn{8}{|c|}{ Rhyme Items } \\
\hline $\mathrm{D} \varnothing \mathrm{b}$ & 845 & 32 & 0 & Dev & 4,235 & 443 & 0 \\
\hline $\mathrm{G} \varnothing \mathrm{b}$ & 658 & 32 & 0 & Gev & 1,710 & 443 & 0 \\
\hline $\mathrm{L} ø \mathrm{~b}$ & 2,045 & 32 & 0 & $\mathrm{Kev}$ & 617 & 443 & 0 \\
\hline $\mathrm{K} \varnothing \mathrm{b}$ & 205 & 32 & 0 & Bev & 9,223 & 443 & 0 \\
\hline $\mathrm{R} \emptyset \mathrm{b}$ & 1,517 & 32 & 0 & Tev & 5,139 & 443 & 0 \\
\hline Møb & 839 & 32 & 0 & Lev & 4,815 & 443 & 0 \\
\hline Gub & 2,024 & 423 & 52 & Tiv & 6,163 & 1,080 & 0 \\
\hline Kub & 3,866 & 423 & 4 & Div & 3,039 & 1,080 & 0 \\
\hline Lub & 1,953 & 423 & 7 & Kiv & 2,198 & 1,080 & 0 \\
\hline Rub & 1,700 & 423 & 0 & Piv & 1,480 & 1,080 & 0 \\
\hline Fub & 5,050 & 2,107 & 3 & Riv & 2,322 & 1,080 & 0 \\
\hline Mub & 2,271 & 423 & 0 & Liv & 4,367 & 1,080 & 0 \\
\hline \multicolumn{8}{|c|}{ Consonant Frame Items } \\
\hline $\mathrm{Kab}$ & 6,311 & 128 & 10 & Bov & 4,992 & 758 & 0 \\
\hline $\mathrm{Keb}$ & 617 & 430 & 0 & Biv & 3,458 & 1,080 & 0 \\
\hline Kob & 11,622 & 1,035 & 0 & Buv & 2,617 & 135 & 0 \\
\hline Kåb & 177 & 5 & 0 & Båv & 514 & 10 & 0 \\
\hline Kib & 2,198 & 290 & 0 & $\mathrm{Bav}$ & 6,583 & 674 & 0 \\
\hline Kub & 3,866 & 423 & 4 & $\mathrm{~B} \emptyset \mathrm{v}$ & 911 & 822 & 0 \\
\hline Pib & 1,480 & 290 & 0 & Teb & 5,139 & 430 & 0 \\
\hline $\mathrm{Pab}$ & 4,633 & 128 & 0 & $\mathrm{Tab}$ & 3,397 & 128 & 0 \\
\hline $\mathrm{Peb}$ & 3,742 & 430 & 0 & Tib & 6,163 & 290 & 0 \\
\hline Påb & 1,273 & 5 & 0 & Tåb & 258 & 5 & 0 \\
\hline Pob & 3,568 & 1,035 & 0 & Tob & 4,314 & 1,035 & 2 \\
\hline Pub & 1,234 & 423 & 0 & Tub & 1,880 & 423 & 3 \\
\hline \multicolumn{8}{|c|}{ Distinct Items } \\
\hline Vur & 256 & 1,494 & 0 & $\mathrm{~K} \varnothing \mathrm{f}$ & 205 & 9 & 0 \\
\hline $\mathrm{Nev}$ & 3,268 & 443 & 0 & $\mathrm{Bis}$ & 3,458 & 2,511 & 0 \\
\hline Kon & 11,622 & 6,537 & 0 & $\mathrm{Sev}$ & 7,016 & 443 & 0 \\
\hline Sid & 4,273 & 2,107 & 5 & Dav & 3,012 & 674 & 0 \\
\hline Båp & 514 & 1,019 & 0 & Gef & 1,710 & 805 & 0 \\
\hline $\mathrm{Kad}$ & 6,311 & 1,854 & 0 & Råm & 790 & 198 & 0 \\
\hline Gup & 2,024 & 253 & 0 & $\mathrm{~T} \varnothing \mathrm{b}$ & 747 & 32 & 0 \\
\hline $\mathrm{D} \varnothing \mathrm{g}$ & 845 & 100 & 0 & Kuv & 3,866 & 135 & 0 \\
\hline Fad & 5,473 & 1,854 & 0 & Reb & 12,398 & 430 & 0 \\
\hline Hud & 2,514 & 1,025 & 0 & $\mathrm{Rab}$ & 3,448 & 128 & 0 \\
\hline Mir & 6,242 & 577 & 0 & $\mathrm{Tad}$ & 3,397 & 1,854 & 0 \\
\hline Def & 4,235 & 443 & 0 & $\mathrm{Bid}$ & 3,458 & 2,107 & 0 \\
\hline \multicolumn{8}{|c|}{ Similar Nonrhyme Items } \\
\hline Fab & 5,473 & 128 & 0 & $\mathrm{Nef}$ & 3,268 & 805 & 0 \\
\hline Laf & 6,354 & 2,565 & 0 & Rel & 12,398 & 3,243 & 1 \\
\hline Sav & 7,820 & 674 & 0 & Neg & 3,268 & 570 & 0 \\
\hline Tar & 3,397 & 3,730 & 0 & Bes & 9,223 & 11,798 & 0 \\
\hline Sap & 7,820 & 1,523 & 0 & Feg & 4,128 & 570 & 0 \\
\hline Gam & 3,277 & 987 & 0 & Tep & 5,139 & 384 & 0 \\
\hline Gåf & 329 & 1 & 0 & Miv & 6,242 & 1,080 & 0 \\
\hline Håd & 1,241 & 393 & 0 & Fid & 5,050 & 2,107 & 0 \\
\hline Fåp & 147 & 1,019 & 0 & Kir & 2,198 & 577 & 0 \\
\hline Råg & 790 & 151 & 0 & $\mathrm{Vib}$ & 5,294 & 290 & 0 \\
\hline Dår & 137 & 754 & 0 & Bim & 3,458 & 549 & 0 \\
\hline Tåk & 258 & 152 & 0 & Tif & 6,163 & 108 & 0 \\
\hline
\end{tabular}

Note-Columns 2, 3, 6, and 7 show the number of different word forms with the biphone in the defined position. $\mathrm{CV}$, consonant-vowel sounds as initials of words; $\mathrm{VC}$, vowel-consonant sounds as finals of words; CVCC, occurrences of the item when written with a double end consonant.

(Manuscript received January 24, 2003;

revision accepted for publication September 18, 2003.) 